\title{
MAXIMUM PRINCIPLES IN THE POTENTIAL THEORY
}

\author{
MASANORI KISHI
}

To Professor Kinjiro Kunugi on the occasion of his 60th birthday

\section{Introduction}

Ninomiya, in his thesis [13] on the potential theory with respect to a positive symmetric continuous kernel $G$ on a locally compact Hausdorff space $\Omega$, proves that $G$ satisfies the balayage (resp. equilibrium) principle if and only if $G$ satisfies the domination (resp. maximum) principle. He starts from the Gauss-Ninomiya variation and shows that for any given compact set $K$ in $\Omega$ and any positive upper semi-continuous function $u$ on $K$, there exists a positive measure $\mu$ on $K$ such that its potential $G \mu$ is $\leq u$ on the support of $\mu$ and $G \mu \geq u$ on $K$ almost everywhere with respect to any positive measure with finite energy.

His method can not be applied to non-symmetric kernels, because for those kernels the Gauss-Ninomiya variation is useless in its original form.

In this paper we shall prove that the above existence theorem is valid for non-symmetric kernels under certain additional conditions-separability of a compact set $K$ and the continuity principle for adjoint kernels. We first prove it in a reduced form on a compact space consisting of a finite number of points and then extend it to a kernel on a locally compact Hausdorff space.

Using our existence theorem, we shall prove that if $G$ and its adjoint $\check{G}$ satisfy the continuity principle, then $G$ satisfies the balayage (resp. equilibrium) principle when and only when $G$ satisfies the domination (resp. maximum) principle. It will be also shown that $G$ satisfies the balayage principle if and only if $\breve{G}$ does. Other maximum principles which are closely connected with the domination and maximum principles will be dealt with also. We shall give an answer to a question, raised by Deny, which concerns with the complete maximum principle.

A summary of this paper was published in [11].

Received July 7, 1963. 


\section{Definitions}

Let $\Omega$ be a locally compact Hausdorff space and $G(x, y)$ be a positive lower semi-continuous kernel on $\Omega$, that is, $G(x, y)$ is defined on the product space $\Omega \times \Omega$ and it is positive and lower semi-continuous as a function on $\Omega \times \Omega$. The kernel $\breve{G}$ defined by $\breve{G}(x, y)=G(y, x)$ is called the adjoint kernel of $G$. If $G \equiv \check{G}, G$ is called symmetric. For a given positive measure $\mu$, the potential $G \mu(x)$ and the adjoint potential $\check{G} \mu(x)$ are defined by

$$
G \mu(x)=\int G(x, y) d \mu(y) \text { and } \breve{G} \mu(x)=\int \breve{G}(x, y) d \mu(y)
$$

respectively. The $G$-energy of $\mu$ is defined by $\int G \mu(x) d \mu(x)$.

We denote by $\mathfrak{M}_{0}$ the family of all positive measures $\mu$ such that its support, denoted by $S_{\mu}$, is compact, and by $\varepsilon_{0}=\mathscr{E}_{0}(G)$ the family of all positive measures in $\mathfrak{M}_{0}$ with finite $G$-energy. Evidently $\mathfrak{E}_{0}(G)=\mathfrak{E}_{0}(\check{G})$. We say that a property holds $G$-p.p.p. on a subset $X \subset \Omega$ when it holds on $X$ almost everywhere with respect to any $\mu$ in $\xi_{0}$. A property holds $G$-p.p.p. on $X$ if and only if it holds $\check{G}$-p.p.p. on $X$. We say that $G$ satisfies the continuity principle when for any $\mu \in \mathfrak{M}_{0}$, the following implication holds:

[the restriction of $G \mu(x)$ to $S_{\mu}$ is finite and continuous]

$\Rightarrow[G \mu(x)$ is finite and continuous in the whole space $\Omega]$.

A seqnence $\left\{\mu_{n}\right\}$ of positive measures is said to converge vaguely to a positive measure $\mu$ when for any finite continuous function $f$ with compact support, $\int f(x) d \mu_{n}(x) \rightarrow \int f(x) d \mu(x)$.

\section{Chapter I. Existence theorem}

1. In this chapter we shall prove the following existence theorem.

Theorem I.1. Let $G$ be a positive lower semi-continuous kernel on $\Omega$ such that its adjoint kernel $\breve{G}$ satisfies the continuity principle. If $K$ is a compact separable subset of $\Omega$ and $u(x)$ is a positive finite upper semi-continuous function on $K$, then there exists a positive measure $\mu$, supported by $K$, such that

$$
\begin{array}{ll}
G \mu(x) \geq u(x) & \text { G-p.p.p. on } K, \\
G \mu(x) \leq u(x) & \text { everywhere on } S_{\mu} .
\end{array}
$$

For symmetric kernels this is well-known. It is verified by using the 
Gauss-Ninomiya variation (cf. Frostman [7], Kametani [9], Ninomiya [13]).

Theorem I. 1 follows immediately from

Theorem I.2. Let $G$ be a positive lower semi-continuous kernel on a compact separable space $\Omega$ such that its adjoint $\breve{G}$ satisfies the continuity principle. If $u(x)$ is a positive finite upper semi-continuous function in $\Omega$, then there exists a positive measure $\mu$ such that

$$
\begin{array}{ll}
G \mu(x) \geq u(x) & \text { G-p.p.p. in } \Omega, \\
G \mu(x) \leq u(x) & \text { on } S \mu .
\end{array}
$$

Therefore it is sufficient to prove Theorem I. 2 and we suppose, throughout this chapter, that $\Omega$ is compact separable, so that it is metriable.

2. First we consider the case that $\Omega$ consists of a finite number of points and reduce Theorem I. 2 to the following

Theorem I.3. Given positive finite numbers $a_{k i}$ and $u_{k}(k, i=1,2, \ldots, n)$, there exist non-negative finite numbers $t_{1}, t_{2}, \ldots, t_{n}$ such that

$$
\begin{array}{ll}
\sum_{i=1}^{n} a_{k i} t_{i} \geq u_{k} & \text { for } k=1,2, \ldots, n, \\
\sum_{i=1}^{n} a_{j i} t_{i}=u_{j} & \text { for every } j \text { such that } t_{j} \neq 0 .
\end{array}
$$

Let $R_{+}^{n}$ be the convex cone in the $n$-dimensional Euclidean space $R^{n}$, consisting of all points $\left(u_{1}, u_{2}, \ldots, u_{n}\right)$ with every $u_{i} \geq 0$. We denote, for $i=1$, $2, \ldots, n$, by $A_{i}$ the point $\left(a_{1 i}, a_{2 i}, \ldots, a_{n i}\right)$ in $R_{+}^{n}$ and, for $m(1 \leq m \leq n)$ positive integers $1 \leq i_{1}<i_{2}<\cdots<i_{m} \leq n$, we denote by $C_{m ; i 1 i 2 \ldots i m}$ the set of all points $\left(u_{1}, u_{2}, \ldots, u_{n}\right) \in R_{+}^{n}$ such that

$$
\begin{aligned}
& u_{k}=\sum_{p=1}^{m} a_{k i_{p}} t_{p} \quad \text { for } k=i_{1}, i_{2}, \ldots, i_{m} \\
& 0 \leq u_{j} \leq \sum_{p=1}^{m} a_{j i_{p}} t_{p} \quad \text { for } j \neq i_{1}, i_{2}, \ldots, i_{m}
\end{aligned}
$$

where $t_{p} \geq 0$ and $\sum t_{p}=1$. The set $C_{m ; i_{1} i_{2} \ldots i_{m}}$ is a convex set on a hyperplane in $R^{n}$, which is parallel to the $\boldsymbol{u}_{j}$-axes $\left(j \neq i_{1}, i_{2}, \ldots, i_{m}\right)$ if $m<n$. We put .

$$
C=C\left[A_{1}, A_{2}, \ldots, A_{n}\right]=\bigcup_{m=1}^{n} \bigcup_{1 \leq i_{1}<i_{2}<\cdots<i_{m} \leq n} C_{m ; i_{1} i_{2} \ldots i_{m}},
$$

and we shall call it the roof determined by $\left\{A_{1}, A_{2}, \ldots, A_{n}\right\}$. 
Now let $S^{n-1}$ be the unit sphere in $R^{n}$ and put $S_{+}^{n-1}=S^{n-1} \cap R_{+}^{n}$, and consider the projection $\tau^{n}$ from the origin 0 to $S_{+}^{n-1}$ in $R_{+}^{n}$. Then we have

Lemma I.1. The projection $\tau^{n}$ maps the roof $C$ onto $S_{+}^{n-1}(n \geq 2)$.

We shall give a proof of this lemma at the next section. Here we prove Theorem I. 3 by using it. For any given point $U=\left(u_{1}, u_{2}, \ldots, u_{n}\right)$ in $R_{+}^{n}$, we have, on the roof $C$ determined by $\left\{A_{1}, A_{2}, \ldots, A_{n}\right\}$, a point $U^{\prime}=\left(u_{1}^{\prime}, u_{2}^{\prime}, \ldots\right.$, $u_{n}^{\prime}$ ) which is on the straight line passing $U$ and the origin 0 . Suppose that $U^{\prime}$ is on $C_{m ; i_{1} i_{2} \ldots i_{m}}$. Then there exist $m$ non-negative $t_{i_{1}}^{\prime}, t_{i_{2}}^{\prime}, \ldots, t_{i_{m}}^{\prime}$ such that

$$
\begin{aligned}
& t_{i_{1}}^{\prime}+t_{i_{2}}^{\prime}+\cdots+t_{i_{m}}^{\prime}=1 \\
& a_{k i_{1}} t_{i_{1}}^{\prime}+a_{k i_{2}} t_{i_{2}}^{\prime}+\cdots+a_{k i_{m}} t_{i_{m}}^{\prime}=u_{k}^{\prime} \quad \text { for } k=i_{1}, i_{2}, \ldots, i_{m} \text {, } \\
& a_{j i_{1}} t_{i_{1}}^{\prime}+a_{j i_{2}} t_{i_{2}}^{\prime}+\cdots+a_{j i_{m}} t_{i_{m}}^{\prime} \geq u_{j}^{\prime} \quad \text { for } j \neq i_{1}, i_{2}, \ldots, i_{m} \text {. }
\end{aligned}
$$

Since $U^{\prime}$ is on the straight line passing $U$ and 0 , there exists a positive number $\alpha$ such that $\alpha u_{k}^{\prime}=u_{k}(1 \leq k \leq n)$. Therefore putting $t_{k}=\alpha t_{k}^{\prime}$ for $k=i_{1}, i_{2}, \ldots$, $i_{m}$ and $t_{j}=0$ for $j \neq i_{1}, i_{2}, \ldots, i_{m}$, we have

$$
\begin{array}{ll}
a_{k 1} t_{1}+a_{k 2} t_{2}+\cdots+a_{k n} t_{n}=u_{k} & \text { for } k \text { with } t_{k} \neq 0, \\
a_{j 1} t_{1}+a_{j 2} t_{2}+\cdots+a_{j n} t_{n} \geq u_{j} & \text { for } j=1,2, \ldots, n .
\end{array}
$$

3. Proof of Lemma I.1. *) We shall prove it by induction. We can easily verify that the statement is true for $n=2$. Let us suppose that the statement is true for $n-1$ and put

$$
A_{i}^{(k)}=\left(a_{1 i}, \ldots, a_{k-1 i}, 0, a_{k+1 i}, \ldots, a_{n i}\right)
$$

for $k, i=1,2, \ldots, n$. Then $(n-1)$ points $A_{1}^{(k)}, \ldots, A_{k-1}^{(k)}, A_{k+1}^{(k)}, \ldots, A_{n}^{(k)}$ are in $R_{+}^{n} \cap\left\{u_{k}=0\right\}$ and

$$
C \cap\left\{u_{k}=0\right\}=I_{k}\left(C\left[A_{1}^{(k)}, \ldots, A_{k-1}^{(k)}, A_{k+1}^{(k)}, \ldots, A_{n}^{(k)}\right]\right),
$$

where $I_{k}$ is the injection which maps $R^{n-1}$ to $R^{n} \cap\left\{\boldsymbol{u}_{k}=0\right\}$. Hence by our assumption the projection $\tau_{k}^{n-1}$ ( = the restriction of $\tau^{n}$ to $I_{k}\left(R^{n-1}\right)$ ) maps $C \cap\left\{u_{k}=0\right\}$ onto $S_{+}^{n-1} \cap\left\{u_{k}=0\right\}$.

On the other hand we have a homeomorphism $h$ which transforms $S_{+}^{n-1}$ onto the $(n-1)$ dimensional ball $B^{n-1}$ in such a way that $\bigcup_{k=1}^{n} S_{+}^{n-1} \cap\left\{u_{k}=0\right\}$ is transformed onto the boundary $\partial B^{n-1}$ of $B^{n-1}$. Thus we have a continuous

*) This proof has been done in collaboration with Prof. N. Shimada. 
mapping $f=h \circ \tau^{n}: C \rightarrow B^{n-1}$ which maps $\bigcup_{k=1}^{n} C \cap\left\{u_{k}=0\right\}$ onto $\partial B^{n-1}$.

Next we construct a continuous onto mapping $g: B^{n-1} \rightarrow C$ which maps $\partial B^{n-1}$ onto $\bigcup_{k=1}^{n} C \cap\left\{u_{k}=0\right\}$. Let $B^{\prime}$ be an $(n-1)$ dimensional ball of radius $1 / 2$, and $B_{1}, B_{2}, \ldots, B_{n}$ be linearly independent $n$ points on the boundary $\partial B^{\prime}$ of $B^{\prime}$. We take a concentric ball $B$ of radius 1 and we project $B_{1}, \ldots, B_{k-1}$, $B_{k+1}, \ldots, B_{n}$ from $B_{k}$ onto the boundary $\partial B$ of $B$. We denote $B_{i}^{(k)}$ the image of $B_{i}$. We can decompose $\partial B$ into simplexes or cells with vertices $B_{i}^{(k)}$, and we can form a cell complex as follows. Take $(n-1)$ points $B_{k}^{(1)}, \ldots, B_{k}^{(k-1)}$, $B_{k}^{(k+1)}, \ldots, B_{k}^{(n)}$. By these points the simplex $\Delta_{1 ; k}$ is determined. Next take $2(n-2)$ points $B_{j}^{(i)}\left(i \neq k_{1}, k_{2}, j=k_{1}, k_{2}\right)$. Then the cell $A_{2} ; k_{1} k_{2}$ is determined by simplexes with vertices $B_{k_{1}}^{(i)}$ and $B_{k_{2}}^{(i)}\left(i \neq k_{1}, k_{2}\right)$. We continue this process. In general, take $m(n-m)$ points $B_{j}^{(i)}\left(i \neq k_{1}, \ldots, k_{m, j}=k_{1}, \ldots, k_{m}\right)$. Then the cell $\Delta_{m} ; k_{1} \ldots k_{m}$ is determined by simplexes with vertices of these points. With these $\partial B$ is decomposed into cells. The number of these cells is $2\left(2^{n-1}-1\right)$.

Next we decompose $\partial B^{\prime}$ into simplexes with vertices $B_{1}, B_{2}, \ldots, B_{n}$. Using the faces of these simplexes and the cell $\Delta_{m ; k_{1} \ldots k_{m}}$ on $\partial B$, we can obtain a decomposition of $\left(B \cap \mathscr{C} B^{\prime}\right) \cup \partial B^{\prime}$ into cells $D_{m_{;} k_{1} \ldots k_{m}}$ such that $D_{m_{i} k_{1} \ldots k_{m}} \cap$ $\partial B=\Delta_{m} ; k_{1} \ldots k_{m}$ and $D_{m ; k_{1} \ldots k_{m}} \cap \partial B^{\prime}=$ simplex with vertices $B_{k_{1}}, \ldots, B_{k_{m}}$. Thus we obtain the decomposition of $B: D_{m ; k_{1} \ldots k_{m}}\left(1 \leq m \leq n-1,1 \leq k_{1}<\cdots<k_{m}\right.$ $\leq n)$ and a simplex $B^{\prime}$ with vertices $B_{1}, B_{2}, \ldots, B_{n}$. The number of these sets is $2^{n}-1$.

Now we construct a continuous onto mapping $g: B \rightarrow C$ which transforms $\partial B$ onto $\bigcup_{k=1}^{n} C \cap\left\{u_{k}=0\right\}$.

At the first step we define a continuous mapping $g_{0}$ from the simplex $B^{\prime}$ with vertices $B_{1}, B_{2}, \ldots, B_{n}$ onto $C_{n ; 12 \ldots n}$ such that the simplex with vertices $B_{i_{1}}, \ldots, B_{i_{m}}$ is transformed onto the simplex with vertices $A_{i_{1}}, \ldots, A_{i_{m}}$ for $1 \leq i_{1}<\cdots<i_{m} \leq n$. At the second step we define a homeomorphism $g_{1 ; k}$ from $D_{1 ; k}$ onto $C_{1 ; k}$ such that $B_{k}$ and $B_{k}^{(i)}$ are transformed to $A_{k}$ and $A_{k}^{(i)}(i \neq k)$ respectively, and $\Delta_{1 ; k}$ to $\bigcup_{i=1}^{n} C_{1 ; k} \cap\left\{u_{i}=0\right\}$. Nex twe define a continuous mapping $g_{2 ; k_{1} k_{2}}: D_{2 ; k_{1} k_{2} \rightarrow C_{2} ; k_{1} k_{2}}$ such that $g_{2 ; k_{1} k_{2}}=g_{1 ; k_{i}}$ on the face with vertices $B_{k_{i}}^{(j)}$ $\left(i=1,2 ; j \neq k_{1}, k_{2}\right)$ and $g_{2 ; k_{1} k_{2}}=g_{0}$ on the face on $\partial B^{\prime}$ with vertices $B_{k_{1}}, B_{k_{2}}$ and $A_{2} ; k_{1} k_{2}$ is mapped onto $\bigcup_{i=1}^{n} C_{2 ; k_{1} k_{2}} \cap\left\{u_{i}=0\right\}$. We continue this process, and we obtain continuous mappings $g_{m ; k_{1} \ldots k_{m}}: D_{m ; k_{1} \ldots k_{m}} \rightarrow C_{m} ; k_{1} \ldots k_{m}(1 \leq m \leq n-1$, 
$1 \leq k_{1}<\cdots<k_{m} \leq n$ ) such that the mapping $g$, defined by

$$
g= \begin{cases}g_{m} ; k_{1} \ldots k_{m} & \text { on } D_{m ; k_{1} \ldots k_{m}} \\ g_{0} & \text { on } B^{\prime},\end{cases}
$$

is a continuous onto mapping $B \rightarrow C$ which maps $B$ onto $\bigcup_{i=1}^{n} C \cap\left\{u_{i}=0\right\}$.

Then we consider the composed continuous mapping $\phi=f \circ g$. This transfoms the $(n-1)$ dimensional ball $B^{n-1}$ to itself and the boundary $\partial B^{n-1}$ onto $\partial B^{n-1}$. As easily seen, the degree of the restriction of $\psi$ on $\partial B^{n-1}$ is 1 (resp. -1 ) if it is sense preserving (resp. sense reversing). Hence by Kronecker's existence theorem (cf. [1] p. 467) $\psi$ is an onto mapping. Therefore $\tau^{n}$ is an onto mapping. This completes the proof.

4. Now we go back to a compact separable space $\Omega$. When $G(x, y)$ is finite continuous everywhere in $\Omega \times \Omega$, we shall say that $G$ is a finite continuous kernel. For later use we state some properties on finite continuous kernels.

Lemma I.2. Let $G$ be a finite continuous kernel and $\left\{\mu_{\alpha}\right\}(\alpha \in A)$ be a family of positive measures such that $\int d \mu_{\alpha} \leq M<+\infty$. Then $G \mu_{\alpha}(x)(\alpha \in A)$ is equi-continuous in $\Omega$.

This is evident, since $G(x, y)$ is finite and continuous on $\Omega \times \Omega$.

Lemma I.3. Let $G$ be a finite continuous kernel. If $\left\{x_{n}\right\}$ converges to $x_{0}$ and a sequence $\left\{\mu_{n}\right\}$ of positive measures converges vaguely to $\mu_{0}$, then

$$
G \mu_{0}\left(x_{0}\right)=\lim G_{\mu_{n}}\left(x_{n}\right) .
$$

Proof. By the preceding lemma, for any positive $\varepsilon$, there exists a neighborhood $\omega\left(x_{0}\right)$ of $x_{0}$ such that

$$
\left|G \mu_{m}\left(x_{0}\right)-G \mu_{m}(x)\right|<\varepsilon / 2 \quad \text { for any } x \in \omega\left(x_{0}\right) \text { and } m .
$$

Therefore there exists $n_{1}$ such that for any $n \geq n_{1}$

$$
\left|G_{\mu_{n}}\left(x_{0}\right)-G \mu_{n}\left(x_{n}\right)\right|<\varepsilon / 2 \text {. }
$$

On the other hand, by the continuity of $G\left(x_{0}, y\right)$ and the vague convergence of $\left\{\mu_{n}\right\}$ there exists $n_{2}$ such that

$$
\left|G_{\mu_{0}}\left(x_{0}\right)-G \mu_{n}\left(x_{0}\right)\right|<\varepsilon / 2 \quad \text { for any } n \geq n_{2} .
$$

Consequently for any $n \geq \max \left(n_{1}, n_{2}\right)$ 


$$
\left|G \mu_{0}\left(x_{0}\right)-G \mu_{n}\left(x_{n}\right)\right|<\varepsilon .
$$

Theorem I.4. Assume that $G$ is a finite continuous kernel on a compact separable space $\Omega$. If $u(x)$ is positive finite continuous on $\Omega$, then there exists a positive measure $\mu$ with properties:

$$
\begin{array}{ll}
G \mu(x) \geq u(x) & \text { on } \Omega \\
G \mu(x)=u(x) & \text { on } S \mu .
\end{array}
$$

Proof. Let $D$ be a dense subset of $\Omega$ which consists of countably many points $x_{n}(n=1,2, \ldots)$ and $D_{n}$ be the subset $\left\{x_{1}, x_{2}, \ldots, x_{n}\right\} . \quad$ By Theorem I. 3 , there exists a positive measure $\mu_{n}$ on $D_{n}$ such that

$$
\begin{aligned}
& G \mu_{n}(x) \geq u(x) \quad \text { on } \quad D_{n}, \\
& G \mu_{n}(x)=u(x) \text { on } S \mu_{n} .
\end{aligned}
$$

Denoting by $\alpha$ and $\beta \min { }_{\Omega \times \Omega} G(x, y)$ and $\max _{\Omega} u(x)$ respectively, we have

$$
\alpha \int d \mu_{n} \leq \int G(x, y) d \mu_{n}(y)=G \mu_{n}(x)=u(x) \leq \beta .
$$

Therefore the total masses of $\mu_{n}$ are bounded and a subsequence of $\left\{\mu_{n}\right\}$ converges vaguely to positive measure $\mu$. Without loss of generality we may suppose that $\left\{\mu_{n}\right\}$ itself converges vaguely to $\mu$. This $\mu$ is what we want. In fact, let $x$ be a point and $\varepsilon$ be a positive number. Then by Lemma I. 2 and the continuity of $u(x)$, there exists a neighborhood $\omega$ of $x$ such that

$$
\begin{array}{ll}
G \mu_{n}(x)+\varepsilon / 3>G \mu_{n}\left(x^{\prime}\right) & \text { for any } x^{\prime} \in \omega \text { and any } n, \\
u\left(x^{\prime}\right)+\varepsilon / 3>u(x) & \text { for any } x^{\prime} \in \omega .
\end{array}
$$

Since $\left\{\mu_{n}\right\}$ converges vaguely to $\mu$,

$$
G \mu(x)+\varepsilon / 3>G \mu_{n}(x) \quad \text { for any sufficiently large } n .
$$

$D$ being lense in $\Omega$, for any sufficiently large $n, D_{n} \cap \omega \neq \varnothing$ and

$$
G \mu_{n}\left(x^{\prime}\right) \geq u\left(x^{\prime}\right) \quad \text { for any } x^{\prime} \in D_{n} \cap \omega .
$$

By (1), (2), (3) and (4), $G \mu(x)+\varepsilon>u(x)$ and hence $G \mu(x) \geq u(x)$.

Now let $x$ be a point of $S \mu$. Then a sequence of points $x_{n} \in S_{\mu_{n}}$ must converge to $x$ and

$$
u(x)=\lim u\left(x_{n}\right)=\lim G \mu_{n}\left(x_{n}\right)=G \mu(x)
$$

by Lemma I. 3. This completes the proof. 
Corollary. Assume the same for $G$ and $\Omega$ as Theorem $I .4$. If $u(x)$ is positive finite upper semi-continuous on $\Omega$, then we have the same conclusion as Theorem I.4.

Proof. Let $\left\{u_{n}\right\}$ be a sequence of positive finite continuous functions such that $u_{n} \searrow u$. By Theorem I. 4, there exist positive measures $\mu_{n}$ such that

$$
\begin{array}{ll}
G \mu_{n}(x) \geq u_{n}(x) & \text { on } \Omega, \\
G \mu_{n}(x)=u_{n}(x) & \text { on } S \mu_{n} .
\end{array}
$$

As is easily seen, the total masses of $\mu_{n}$ are bounded and a subsequence converges vaguely to a positive measure $\mu$. Without loss of generality we may suppose that $\left\{\mu_{n}\right\}$ converges vaguely to $\mu$. Then we have

$$
G \mu(x)=\lim G \mu_{n}(x) \geq \lim u_{n}(x)=u(x)
$$

in $\Omega$. On the other hand, for a point $x$ on $S \mu$, a sequence $\left\{x_{n}\right\}$ of points on $S \mu_{n}$ converges to $x$ and

$$
G \mu(x)=\lim G \mu_{n}\left(x_{n}\right)=\lim u_{n}\left(x_{n}\right) \leq u(x) .
$$

Thus the existence of a wanted positive measure $\mu$ has been shown.

5. Now we consider a kernel $G$ which is not necessarily finite and continuous.

Lemma I.4 (cf. Brelot and Choquet [2], Ohtsuka [16]). Assume that the adjoint kernel $\breve{G}$ satisfies the continuity principle. If a sequence $\left\{\mu_{n}\right\}$ of positive measures converges vaguely to $\mu$, then

$$
\underline{\lim } G \mu_{n}(x)=G \mu(x) \quad \text { G.p.p.p. in } \Omega \text {. }
$$

Proof. Since $\left\{\mu_{n}\right\}$ converges vaguely to $\mu, \underline{\lim } G \mu_{n}(x) \geq G \mu(x)$ everywhere in $\Omega$. Therefore it is sufficient to show that $\lim G \mu_{n}(x) \leq G \mu(x) G$-p.p.p. in $\Omega$. If we deny this, we can find a unit measure $\nu$ with finite $G$-energy such that

$$
\underline{\lim } G \mu_{n}(x)>G \mu(x) \quad \text { on } S \nu .
$$

Since $\int \breve{G} \nu d \nu=\int G \nu d \nu<+\infty, \breve{G} \nu$ is finite continuous on a compact subset $K_{1} \subset S_{\nu}$, and hence by the continuity principle, the potential $G_{\nu_{1}}$ of the restriction of $\nu$ to $K_{1}$ is finite continuous in $\Omega$. Consequently 


$$
\begin{aligned}
\int G \mu d \nu_{1}<\int \underline{\lim } G \mu_{n} d \nu_{1} & <\underline{\lim \int G \mu_{n} d \nu_{1}} \\
& =\lim \int \breve{G} \nu_{1} d \mu_{n}=\int \breve{G} \nu_{1} d \mu=\int G \mu d \nu_{1} .
\end{aligned}
$$

This is a contradiction, which completes the proof.

6. Now we can prove Theorem I.2. Let us take positive finite continuous kernels $G_{n}$ of $\Omega$ such that $G_{n} \nearrow G$. Then we have, by Corollary of Theorem I. 4 , a positive measure $\mu_{n}$ such that

$$
\begin{array}{ll}
G_{n} \mu_{n}(x) \geq u(x) & \text { on } \Omega, \\
G_{n} \mu_{n}(x)=u(x) & \text { on } S \mu_{n} .
\end{array}
$$

The total masses of $\mu_{n}$ are bounded and a subsequence converges vaguely to a positive measure $\mu$. Without loss of generality we may suppose that $\left\{\mu_{n}\right\}$ itself converges vaguely to $\mu$. Then by Lemma I. 4,

$$
G \mu(x)=\underline{\lim } G \mu_{n}(x) \geq \underline{\lim } G_{n} \mu_{n}(x) \geq u(x)
$$

$G$-p.p.p. on $\Omega$. On the other hand, for any point $x$ on $S \mu$, a sequence $\left\{x_{n}\right\}$ $\left(x_{n} \in S \mu_{n}\right)$ converges to $x$ and for any fixed $n_{0}$

$$
\begin{aligned}
u(x) & \geq \varlimsup \lim u\left(x_{n}\right)=\varlimsup \\
& \geq \lim G_{n_{0}} \mu_{n}\left(x_{n}\right)=G_{n_{0}} \mu(x) .
\end{aligned}
$$

Therefore $u(x) \geq G \mu(x)$. Consequently $G \mu(x)$ has all the properties of our theorem. This completes the proof.

Remark. Theorem I. 1 is false, unless we assume the continuity principle for the adjoint kernel; it is shown by a simple counter-example.

\section{Chapter II. Maximum principles}

1. In this chapter we suppose that $\Omega$ is a locally compact Hausdorff space every compact subset of which is separable and that $G$ is a positive lower semi-continuous kernel on $\Omega$ such that $G(x, y)$ is locally bounded at every point $(x, y) \in \Omega \times \Omega$ with $x \neq y$.

Let us start from the following principles.

Let $u(x)$ be a positive finite upper semi-continuous function or a positive lower semi-continuous function which is $\neq+\infty$ in $\Omega$, and $v(x)$ be a positive $\neq+\infty$ function in $\Omega$ such that $u(x) \leq v(x)$ everywhere. 
(I) $(u, v)$-relative equilibrium principle. For any compact set $K$, there exists a positive measure $\mu \in \mathfrak{M}_{0}$, supported by $K$, such that

$$
\begin{array}{ll}
G \mu(x) \geq u(x) & G \text {-p.p.p. on } K, \\
G \mu(x) \leq v(x) & \text { in } \Omega .
\end{array}
$$

(II) $(u, v)$-relative domination principle. For a positive measure $\mu \in \mathfrak{E}_{0}$, an inequality $G \mu(x) \leq u(x)$ on $S \mu$ implies the inequality $G \mu(x) \leq v(x)$ in $\Omega$.

Choquet and Deny [4] introduced these principles with $u=v$. Ohtsuka [16] dealt with similar principles.

Theorem II.1. Let $G$ and $\breve{G}$ satisfy the continuity principle. Then $G$ satisfies the $(u, v)$-relative equilibrium principle if and only if $G$ satisfies the $(u, v)$-relative domination principle.

Proof. (I) $\rightarrow$ (II). Let $G$ satisfy the $(u, v)$-relative equilibrium principle and let $\mu$ be a positive measure in $\xi_{0}$ such that $G \mu \leq u$ on $S_{\mu}$. Take a point $x$ in $\Omega-S \mu$ and a sequence of positive finite continuous functions $f_{n}$ on $S \mu$ such that $f_{n} \nearrow \breve{G} \varepsilon_{x}$, where $\varepsilon_{x}$ is the unit measure such that $\int f d \varepsilon_{x}=f(x)$ for any finite continuous function $f$ in $\Omega$. Then by the existence theorem (Theorem I.1) we have positive measures $\nu_{n}$ on $S_{\mu}$ such that

$$
\begin{array}{ll}
\check{G} \nu_{n} \geq f_{n} & G \text {-p.p.p. on } S \mu, \\
\check{G} \nu_{n} \leq f_{n} & \text { on } S \nu_{n} \subset S \mu .
\end{array}
$$

From the first inequality it follows that

$$
\begin{aligned}
& G \mu(x)=\int G \mu d \varepsilon_{x}=\int \check{G} \varepsilon_{x} d \mu=\lim \int f_{n} d \mu
\end{aligned}
$$

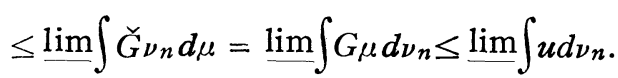

Let $\mu_{n}^{\prime}$ be $(u, v)$-relative equilibrium measures on $S \nu_{n}$. Then each $\mu_{n}^{\prime}$ is supported by $S \nu_{n}$ and $G \mu_{n}^{\prime} \geq u G$-p.p.p. on $S \nu_{n}$ and $G \mu_{n}^{\prime} \leq v$ in $\Omega$. Hence

$$
\begin{aligned}
\int u d \nu_{n} \leq \int G \mu_{n}^{\prime} d \nu_{n} & =\int \breve{G} \nu_{n} d \mu_{n}^{\prime} \leq \int f_{n} d \mu_{n}^{\prime} \leq \int \breve{G} \varepsilon_{x} d \mu_{n}^{\prime} \\
& =\int G \mu_{n}^{\prime} d \varepsilon_{x} \leq \int v d \varepsilon_{x}=v(x),
\end{aligned}
$$

where the first inequality follows from the fact that $\int G_{\nu_{n}} d \nu_{n} \leq \int f_{n} d \nu_{n}<+\infty$. 
Consequently $\overline{\lim } \int u d \nu_{n} \leq v(x)$ and $G \mu(x) \leq v(x)$.

(II) $\rightarrow$ (I). Let $G$ satisfy the $(u, v)$-relative domination principle and $K$ be a compact set. We can take a sequence $\left\{u_{n}\right\}$ of positive finite upper semicontinuous functions with $u_{n} \nearrow u$ on $K$. Then by the existence theorem there exists a sequence $\left\{\mu_{n}\right\}$ of positive measures, supported $K$, such that

$$
\begin{array}{ll}
G \mu_{n}(x) \geq u_{n}(x) & G \text {-p.p.p. on } K, \\
G \mu_{n}(x) \leq u_{n}(x) & \text { on } S \mu_{n} .
\end{array}
$$

By the last inequality, $\mu_{n}$ belongs to $E_{0}$ and by the $(u, v)$-relative domination principle,

$$
G \mu_{n}(x) \leq v(x) \quad \text { in } \Omega .
$$

From this follows that the total masses of $\mu_{n}$ are bounded and a subsequence converges vaguely to a positive measure $\mu$ supported by $K$. We may suppose that $\mu_{n} \rightarrow \mu$ vaguely. Then

$$
G \mu(x)=\underline{\lim } G \mu_{n}(x) \geq \lim u_{n}(x)=u(x)
$$

$G$-p.p.p. on $K$. Thus $G$ satisfies the $(u, v)$-relative equilibrium principle.

In the following sections we shall give applications of this theorem.

2. Domination principle. First application of the preceding theorem is on the balayage principle.

(III) Balayage principle. For any compact set $K$ and any $\mu \in \mathfrak{M}_{0}$, there exists $\mu^{\prime} \in \mathfrak{M}_{0}$ such that $\mu^{\prime}$ is supported by $K$ and

$$
\begin{array}{ll}
G \mu^{\prime}(x) \leq G \mu(x) & \text { in } \Omega . \\
G \mu^{\prime}(x) \geq G \mu(x) & G \text {-p.p.p. on } K .
\end{array}
$$

This measure $\mu^{\prime}$ is called a $G$-balayaged measure of $\mu$ on $K$.

( IV Domination principle. For a positive measure $\mu \in E_{0}$ and a. positive measure $\nu \in \mathfrak{M}_{0}$, an inequality $G_{\mu}(x) \leq G_{\nu}(x)$ on $S_{\mu}$ implies the same inequality in the whole space $\Omega$.

$(V)$ Elementary domination principle. For a positive measure $\mu \in \mathfrak{E}_{0}$ and a point $x$ not on $S \mu$, an inequality $G \mu(z) \leq G \varepsilon_{x}(z)$ on $S_{\mu}$ implies the same inequality in $\Omega$.

TheоRем II.2. If $G$ and $\breve{G}$ satisfy the continuity principle, then the 
following four statements are equivalent.

(1) $G$ satisfies the balayage principle,

(2) $\breve{G}$ satisfies the balayage principle,

(3) G satisfies the domination principle,

(4) $\breve{G}$ satisfies the domination principle.

Ninomiya [12] first obtained this theorem for symmetric kernels, then Deny [5] followed to show the equivalence between (1) and (4) for strictly increasing diffusion kernels. For regular kernels on a compact space which consists of a finite number of points, Theorem II. 2 was obtained by Choquent and Deny [4].

Proof of Theorem II.2. (1) $\rightarrow(4)$. Let $\mu$ be a positive measure in $\mathbb{F}_{0}$ and $\nu$ be one in $\mathfrak{M}_{0}$ such that $\breve{G} \mu \leq \breve{G} \nu$ on $S \mu$. Take a point $x$ in $\Omega-S_{\mu}$ and a $G$-balayaged measure $\varepsilon_{x}^{\prime}$ of $\varepsilon_{x}$ on $S \mu$. Then

$$
\begin{aligned}
\breve{G} \mu(x) & =\int \breve{G} \mu d \varepsilon_{x}=\int G \varepsilon_{x} d \mu=\int G \varepsilon_{x}^{\prime} d \mu \\
& =\int \check{G} \mu d \varepsilon_{x}^{\prime} \leq \int \check{G} \nu d \varepsilon_{x}^{\prime}=\int G \varepsilon_{x}^{\prime} d \nu \\
& \leq \int G \varepsilon_{x} d \nu=\int \check{G} \nu d \varepsilon_{x}=\check{G} \nu(x),
\end{aligned}
$$

where the third equality, $\int G \varepsilon_{x} d \mu=\int G \varepsilon_{x}^{\prime} d \mu$, follows from the facts that $G \varepsilon_{x}=$ $G \varepsilon_{x}^{\prime} G$-p.p.p. on $S \mu$ and $\mu$ belongs to $\varepsilon_{0}$, so that $G \varepsilon_{x}=G \varepsilon_{x}^{\prime} \mu$-almost everywhere.

$(3) \rightarrow(1)$. Let $G$ satisfy the domination principle and let $\mu$ be a positive measure in $\mathfrak{M}_{0}$. Putting $u(x)=v(x)=G \mu(x)$, we see that for any compact set $K$, there exists a $G$-balayaged measure of $\mu$ on $K$ by Theorem II. 1 . Hence $G$ satisfies the balayage principle.

The implications $(2) \rightarrow(3)$ and $(4) \rightarrow(2)$ are duals of $(1) \rightarrow(4)$ and $(3) \rightarrow(1)$ respectively. This completes the proof.

Remark. This theorem is valid for kernels which are not necessarily locally bounded.

Now we shall give another characterization of the balayage principle, which will be useful in applications.

Theorem II.3. Let $G$ and $\breve{G}$ satisfy the continuity principle. Then the following statements and (1) in Theorem II. 2 are equivalent.

(5) $G$ satisfies the elementary domination principle, 
(6) $\breve{G}$ satisfies the elementary domination principle.

Proof. (1) $\rightarrow(5)$. Let $G$ satisfy the balayage principle. Then by the preceding theorem $G$ satisfies the domination principle, whence $G$ does the elementary domination principle.

$(5) \rightarrow(1)$. Let $G$ satisfy the elementary domination principle and $\mu$ be a positive measure in $\mathfrak{F}_{0}$ and $\nu$ be a positive measure in $\mathfrak{M}_{0}$ such that $\breve{G} \mu \leq \breve{G} \nu$ on $S \mu$. Take a point $x$ in $\Omega-S \mu$. Then we have a $G$-balayaged measure $\lambda$ of $\varepsilon_{x}$ on $S \mu$ by the elementary domination principle and the existence theorem. This $\lambda$ belongs to $\varepsilon_{0}$, since $\int G \lambda d \lambda \leq \int G \varepsilon_{x} d \lambda$ and $G \varepsilon_{x}$ is bounded on $S \mu$. Then

$$
\begin{aligned}
\breve{G} \mu(x) & =\int \breve{G} \mu d \varepsilon_{x}=\int G \varepsilon_{x} d \mu=\int G \lambda d \mu \\
& =\int \breve{G} \mu d \lambda \leq \int \breve{G} \nu d \lambda=\int G \lambda d \nu \\
& \leq \int G \varepsilon_{x} d \nu=\int \check{G} \nu d \varepsilon_{x}=\check{G} \nu(x) .
\end{aligned}
$$

Consequently $\breve{G}$ satisfies the domination principle and $G$ satisfies the balayage principle.

The equivalence between (2) and (6) is dual of the one between (1) and (5). Hence (1) and (6) are equivalent by Theorem II. 2.

This theorem was obtained by Ninomiya [13] for symmetric kernels.

Remark. If $G$ and $\breve{G}$ satisfy the continuity principle, $G$ satisfies the balayage principle when and only when one of the following statements is true :

$G$-balayaged measures of point-masses exist always,

$\breve{G}$-balayaged measures of point-masses exist always.

3. Dilated domination principle. Now let us consider the dilated domination principle after Ohtsuka [16].

(III)' k-dilated balayage principle. For any compact set $K$ and any $\mu \in \mathfrak{M}_{0}$, there exists $\mu^{\prime} \in \mathbb{M}_{0}$ such that $\mu^{\prime}$ is supported by $K$ and

$$
\begin{array}{ll}
G \mu^{\prime}(x) \leq k \cdot G \mu(x) & \text { in } \Omega, \\
G \mu^{\prime}(x) \geq G \mu(x) & G \text {-p.p.p. on } K .
\end{array}
$$

$(I V)^{\prime} k$-dilated domination principle. For a positive measure $\mu \in \xi_{0}$ and a 
positive measure $\nu \in \mathbb{M}_{0}$, an inequality $G_{\mu}(x) \leq G_{\nu}(x)$ on $S \mu$ implies the inequality $G \mu(x) \leq k \cdot G \nu(x)$ in $\Omega$.

$(V)^{\prime} k$-dilated elementary domination principle. For a positive measure $\mu \in \varepsilon_{0}$ and a point $x$ not on $S \mu$, an inequality $G \mu(z) \leq G \varepsilon_{x}(z)$ on $S_{\mu}$ implies the inequality $G \mu(z) \leq k \cdot G \varepsilon_{x}(z)$ in $\Omega$.

Using just the same as in the preceding arguments we obtain

ThEOREM II.4. If $G$ and $\breve{G}$ satisfy the continuity principle, then the following statements are equivalent: $G$ satisfies the $k$-dilated balayage principle, $G$ satisfies the $k$-dilated domination principle, $G$ satisfies the $k$-dilated elementary domination principle, $\breve{G}$ satisfies the $k$-dilated balayage principle, $\breve{G}$ satisfies the $k$ dilated domination principle, $\breve{G}$ satisfies the $k$-dilated elementary domination principle.

4. Maximum principle. Now we consider the equilibrium and maximum principles.

(IV) Equilibrium principle. For any compact set $K$, there exists a positive measure $\mu$, supported by $K$, such that

$$
\begin{array}{ll}
G \mu(x) \leq 1 & \text { in } \Omega, \\
G \mu(x)=1 & G \text {-p.p.p. on } K .
\end{array}
$$

This measure $\mu$ is called a $G$-equilibrium measure of $K$.

(VII) Maximum principle. For a positive measure $\mu \in \mathbb{M}_{0}$, an inequality $G \mu(x) \leq 1$ on $S_{\mu}$ implies the same inequality in $\Omega$.

$(V I I)^{\prime} k$-dilated maximum principle. If $G \mu(x) \leq 1$ on $S_{\mu}$ for $\mu \in \mathfrak{M}_{0}$, then $G \mu(x) \leq k$ in $\Omega$.

The following theorems are immediate consequences of Theorem II. 1.

Theorem II.5. Let $G$ and $G$ satisfy the continuity principle. Then $G$ satisfies the equilibrium principle if and only if $G$ satisfies the maximum principle.

THEOREM II.6. Let $G$ and $\breve{G}$ satisfy the continuity principle. Then $G$ satisfies the k-dilated maximum principle when and only when for any compact set $K$, there exists a positive measure $\mu$, supported by $K$, such that

$$
\begin{array}{ll}
G \mu(x) \geq 1 & \text { G-p.p.p. on } K, \\
G \mu(x) \leq k & \text { in } \Omega .
\end{array}
$$


Remark. Contrary to the preceding equivalence $(1) \leftrightarrow(2)$, $(r$ does not satisfy the equilibrium principle in general, although $G$ satisfies it. The equivalense holds for convolution kernels.

ThEOREM II. 7. Assume that $G$ and $\breve{G}$ satisfy the continuity principle. Then $G$ satisfies the equilibrium principle if and only if the following property [Ц] is fulfilled for the adjoint kernel $\breve{G}$.

[Q] For a positive measure $\mu \in \mathfrak{M}_{0}$ and a point $x$ which is not on $S_{\mu}$, the validity of an inequality $\breve{G} \mu \leq \breve{G} \varepsilon_{x}$ on $S_{\mu}$ implies that $\int d \mu \leq 1$.

Theorem II. 8. Assume that $G$ and $\breve{G}$ satisfy the continuity principle. Then $G$ satisfies the $k$-dilated maximum principle if and only if the following property $[\check{Q}]_{k}$ is fulfilled for the adjoint kernel $\breve{G}$.

$[\check{Q}]_{k}$ for a positive measure $\mu \in \mathfrak{M}_{0}$ and a point $x$ which is not on $S \mu$, the validity of an inequality $\breve{G} \mu \leq \breve{G} \varepsilon_{x}$ on $S_{\mu}$ implies that $\int d \mu \leq k$.

For symmetric kernels Theorems II. 5 and 7 were obtained by Ninomiya [13] and Theorems II. 6 and 8 were by Ohtsuka [16].

We shall prove Theorem II. 8 . Let $G$ satisfy the $k$-dilated maximum principle and $\mu$ be a positive measure in $\mathfrak{M}_{0}$ such that $\breve{G} \mu \leq \breve{G} \varepsilon_{x}$ on $S \mu$ for a point $x$ which is not on $S \mu$. By Theorem II. 6 there exists a positive measure $\nu$ on $S_{\mu}$ such that

$$
\begin{array}{ll}
G \nu \geq 1 & G \text {-p.p.p. on } S_{\mu}, \\
G \nu \leq k & \text { in } \Omega .
\end{array}
$$

Then, $\mu$ being in $\mathfrak{s}_{0}$,

$$
\begin{aligned}
\int d \mu & \leq \int G \nu d \mu=\int \breve{G} \mu d \nu \leq \int \breve{G} \varepsilon_{x} d \nu \\
& =\int G \nu d \varepsilon_{x} \leq k \int d \varepsilon_{x}=k .
\end{aligned}
$$

Conversely we suppose that $\breve{G}$ fulfills the condition $[\breve{Q}]_{k}$ and $\mu$ is a positive measure in $\mathfrak{M}_{0}$ such that $G \mu \leq 1$ on $S_{\ell}$. We take a point $x$ in $\Omega-S_{\mu}$ and a sequence $\left\{u_{n}\right\}$ of finite continuous functions on $S \mu$ such that $u_{n} \nearrow G \varepsilon_{x}$. Then there exist positive measures $\nu_{n}$, supported by $S \mu$, such that

$$
\begin{array}{ll}
G \nu_{n} \geq u_{n} & G \text {-p.p.p. on } S \mu, \\
G \nu_{n} \leq u_{n} & \text { on } S \nu_{n} .
\end{array}
$$


From the last inequality it follows that $\int d_{\nu_{n}} \leq k$. Hence

$$
\begin{aligned}
\check{G} \mu(x) & =\int \breve{G} \mu d \varepsilon_{x}=\int G \varepsilon_{x} d \mu=\lim \int u_{n} d \mu \\
& \leq \underline{\lim } \int G \nu_{n} d \mu=\underline{\lim \int \breve{G} \mu d \nu_{n}} \\
& \leq \underline{\lim \int} \int d_{\nu_{n}} \leq k .
\end{aligned}
$$

5. Complete maximum principle. Here we consider the complete maximum principle and related principles. The complete maximum principle was first introduced by Cartan and Deny [3]. Hunt [8] and many other probabilists investigated it.

(VIII) Complete maximum principle. For a positive measure $\mu \in \mathfrak{E}_{0}$, a positive measure $\nu \in \mathfrak{M}_{0}$ and for a non-negative number $a$, the validity of an inequality $G_{\mu}(x) \leq G \nu(x)+a$ on $S_{\mu}$ implies the validity of the same inequality in $\Omega$.

(IX) Strong maximum principle. If $\mu$ is a positive measure in $\mathfrak{F}_{0}$ and $\nu$ is a positive measure in $\mathfrak{M}_{0}$ such that $G \mu \leq G \nu+a$ on $S_{\mu} \cup S_{\nu}$ with a non-negative number $a$, then the same inequality holds in $\Omega$.

Evidently $G$ satisfies the strong maximum principle, if it does the complete maximum principle. Deny [6] asked a question wether the converse is true under certain hypothesis on regularity. He solved in the affirmative for kernels on a space of a finite number of points. We also give an affirmative answer for our kernels.

Theorem II.9. Let $\breve{G}$ satisfy the continuity principle. Then $G$ satisfies the complete maximum principle if $G$ does the strong maximum principle.

First we prove

Lemma II.1. If $G$ satisfies the maximum principle, then it has the following property: Let $\mu$ be a positive measure in $\mathfrak{M}_{0}$ and $x$ be a point in $\Omega-S_{\mu}$. Then $G(x, x)$ majorizes $G \mu(z)$ for $z \in \Omega$, if $G \varepsilon_{x}(z)$ majorizes $G \mu(z)$ for any $z \in S_{\mu}$.

Proof. We may suppose that $G(x, x)$ is finite. Then by the maximum principle, $G \varepsilon_{x}(z) \leq G(x, x)$ in $\Omega$. Therefore 


$$
\sup _{z \in S \mu} G \mu(z) \leq \sup _{z \in S \mu} G \varepsilon_{x}(z) \leq G(x, x) .
$$

Then again by the maximum principle, $G \mu(z) \leq G(x, x)$ for any $z \in \Omega$.

Theовем II. 10. Let $\breve{G}$ satisfy the continuity principle. Then $G$ satisfies the complete maximum principle if and only if $G$ satisfies the domination and maximum principles.

Proof. It is sufficient to prove that $G$ satisfies the complete maximum principle if it does the domination and maximum principles, since the converse is evident. Let $\mu$ be in $\mathbb{E}_{0}$ and $\nu$ be in $\mathfrak{M}_{0}$ such that $G \mu \leq G \nu+a$ on $S_{\mu}$ with $a \geq 0$. Take a point $x$ in $\Omega-S \mu$. Since $G$ satisfies the domination principle, $\breve{G}$ satisfies it and there exists a $\breve{G}$-balayaged measure $\varepsilon_{x}^{\prime}$ of $\varepsilon_{x}$ on $S_{\mu}$. Let $\lambda$ be a $G$-equilibrium measure of $S \mu$. Because $\varepsilon_{x}^{\prime}$ belongs to $E_{0}$,

$$
\begin{aligned}
\int d \varepsilon_{x}^{\prime} & =\int G \lambda d \varepsilon_{x}^{\prime}=\int \breve{G} \varepsilon_{x}^{\prime} d \lambda \leq \int \breve{G} \varepsilon_{x} d \lambda \\
& =\int G \lambda d \varepsilon_{x} \leq \int d \varepsilon_{x}=1 .
\end{aligned}
$$

Therefore

$$
\begin{aligned}
G \mu(x) & =\int G \mu d \varepsilon_{x}=\int \breve{G} \varepsilon_{x} d \mu=\int \breve{G} \varepsilon_{x}^{\prime} d \mu \\
& =\int G \mu d \varepsilon_{x}^{\prime} \leq \int(G \nu+a) d \varepsilon_{x}^{\prime} \leq \int G \nu d \varepsilon_{x}^{\prime}+a \\
& \leq \int \breve{G} \varepsilon_{x} d \nu+a=\int G \nu d \varepsilon_{x}+a=G \nu(x)+a .
\end{aligned}
$$

Thus $G$ satisfies the complete maximum principle.

Proof of Theorem II.9. By Theorem II. 10 it is sufficient to show that if $G$ satisfies the strong maximum principle, then $G$ fulfills the condition (5) in Theorem II 3. Let $\mu$ be a positive measure in $\mathbb{E}_{0}$ and $x$ be a point not on $S \mu$ such that $G \mu \leq G \varepsilon_{x}$ on $S_{\mu}$. Then by Lemma II. 2, this inequality holds at $x$ also. Hence by the strong maximum principle, it holds everywhere in $\Omega$. Consequently $G$ satisfies the complete maximum principle.

A characterization of the complete maximum principle by the following balayage principle with mass-diminution was given by Deny [6].

$(X)$ Balayage principle with mass-diminution. The balayage principle with the following property: the total mass of the balayaged measure $\mu^{\prime}$ of a 
positive measure $\mu$ with compact support onto a compact set is less than that of $\mu$.

THEOREM II. 11. If $\breve{G}$ satisfies the balayage principle with mass-diminution, then $G$ satisfies the complete maximum principle. The converse is true if $G$ and $\breve{G}$ satisfy the continuity principle and $G$ is regular in the sense of $[10] p .65$.

Proof. Suppose that $\breve{G}$ satisfies the balayage principle with mass-diminution. Let $\mu$ and $\nu$ be positive measures with compact support such that $\int G \mu d \mu$ is finite and $G_{\mu} \leq G_{\nu}+a$ on $S_{\mu}$ with $a \geq 0$. Take a point $x$ in $\Omega-S \mu$. Since $\check{G}$ satisfies the balayage principle with mass-diminution, there exists a $\breve{G}$ balayaged measure $\varepsilon_{x}^{\prime}$ of $\varepsilon_{x}$ on $S_{\mu}$ such that $\int d \varepsilon_{x}^{\prime} \leq 1$. Then by the same computation as in the proof of Theorem II. 10 we have $G \mu(x) \leq G \nu(x)+a$.

Conversely suppose that $G$ satisfies the complete maximum principle. Then by Theorems II. 2 and $5, \breve{G}$ satisfies the balayage principle and $G$ satisfies the equilibrium principle. Let $\mu^{\prime}$ be a $G^{\prime}$-balayaged measure of a positive measure $\mu \in \mathfrak{M}_{0}$ on a compact set $K$, and let $\nu$ be a $G$-equilibrium measure of a $G$ regular compact set $K^{\prime} \supset K$. Then

$$
\int d \mu^{\prime}=\int G \nu d \mu^{\prime}=\int \check{G} \mu^{\prime} d \nu \leq \int \check{G} / \iota d \nu=\int G \nu d \mu \leq \int d \mu .
$$

Consequently $\breve{G}$ satisfies the balayage principle with mass-diminution.

Theorem II. 1 supplies ancther characterization of the complete maximum principle.

(XI) Complete balayage principle. For any positive measure $\mu$ with compact support, any compact set $K$ and any $a \geq 0$, there exists a positive measure $\mu^{\prime}$, supported by $K$, such that

$$
\begin{array}{ll}
G \mu^{\prime}=G \mu+a & G \text {-p.p.p. on } K, \\
G \mu^{\prime} \leq G \mu+a & \text { in } \Omega .
\end{array}
$$

Theorem II. 12. Let $G$ and $\breve{G}$ satisfy the continuity principle. Then $G$ satisfies the complete maximum principle if and only if $G$ satisfies the complete balayage principle.

This follows immediately from Theorem II. 1.

Now we state the dilated principles. 
$(V I I I)^{\prime}\left(k_{1}, k_{2}\right)$-dilated complete maximum principle. For a positive measure $\mu \in \mathfrak{E}_{0}$, a positive measure $\nu \in \mathfrak{M}_{0}$ and a non-negative number $a$, the validity of the inequality $G \mu(x) \leq G \nu(x)+a$ on $S_{\mu}$ implies the inequality $G \mu(x) \leq k_{1} G_{\nu}(x)$ $+k_{2} a$ in $\Omega$.

$(I X)^{\prime}\left(k_{1}, k_{2}\right)$-dilated strong maximum principle. If $\mu$ is a positive measure in $\mathfrak{F}_{0}$ and $\nu$ is a positive measure in $\mathfrak{M}_{0}$ such that $G \mu \leq G \nu+a$ on $S_{\mu} \cup S_{\nu}$ with a non-negative number $a$, then $G \mu \leq k_{1} G \nu+k_{2} a$ in $\Omega$.

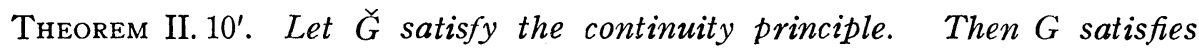
the $\left(k_{1}, k_{1} k_{2}\right)$-dilated complete maximum principle if $G$ satisfies the $k_{1}$-dilated domination principle and the $k_{2}$-dilated maximum principle.

TheOREM II. $9^{\prime}$. Let $\breve{G}$ satisfy the continuity principle. Then G satisfies the $\left(k_{1} k_{2}^{2}, k_{1} k_{2}^{3}\right)$-dilated complete maximum principle if $G$ satisfies the $\left(k_{1}, k_{2}\right)$ dilated strong maximum principle.

6. Weak domination principle. Let us add a few words to the weak domination principle.

(XII) Weak domination principle. If $\mu$ is a positive measure in $\xi_{0}$ and $\nu$ is a positive measure in $\mathfrak{M}_{0}$ such that $G \mu \leq G \nu$ on $S_{\mu} \cup S_{\nu}$, then the same inequality holds in $\Omega$.

TheOREM II. 13. Assume that $G$ and $\breve{G}$ satisfy the continuity principle and that $G$ satisfies the weak domination principle. If $G$ satisfies the maximum principle or $G(x, x)=+\infty$ for any $x \in \Omega$, then $G$ satisfies the domination principle.

Proof. By Theorem II.3, it is sufficient to show that $G$ satisfies the elementary domination principle. Let $\mu$ be a positive measure in $\varepsilon_{0}$ and $x$ be a point in $\Omega-S \mu$ such that $G \mu \leq G \varepsilon_{x}$ on $S \mu$. Then $G \mu(x) \leq G \varepsilon_{x}(x)$ if $G$ satisfies the maximum principle or $G(x, x)=+\infty$. Hence by the weak domination principle, $G \mu \leq G \varepsilon_{x}$ everywhere in $\Omega$. Thus $G$ satisfies the elementary domination principle.

THEOREM II. 13'. If $\check{G}$ satisfies the continuity principle and $G$ satisfies the weak domination principle and the k-dilated maximum principle, then $G$ satisfies the $k^{2}$-dilated domination principle. $k^{2}$ can not be replaced by a smaller number. 
7. Domination principle with respect to $\boldsymbol{N}$. Now let $N$ be another positive lower semi-continuous kernel on $\Omega$, and consider the following principles after Ninomiya $[14,15]$.

(XIII) Balayage principle with respect to $N$. For any compact set $K$ and any positive measure $\mu$ in $\mathfrak{M}_{0}$, there exists a positive measure $\mu^{\prime}$, supported by $K$, such that

$$
\begin{array}{ll}
G \mu^{\prime}(x)=N \mu(x) & G \text {-p.p.p. on } K, \\
G \mu^{\prime}(x) \leq N \mu(x) & \text { in } \Omega .
\end{array}
$$

(XIV) Domination principle with respect to $N$. For any positive measure $\mu$ in $\mathfrak{E}_{0}=\mathfrak{E}_{0}(G)$ and for any positive measure $\nu$ in $\mathfrak{M}_{0}$, the inequality $G \mu(x) \leq$ $N_{\nu}(x)$ on $S_{\mu}$ implies the same inequality in $\Omega$.

( $X V)$ Elementary domination principle with respect to $N$. For any positive measure $\mu$ in $\mathfrak{E}_{0}=\mathfrak{E}_{0}(G)$ and a point $x$ not on $S \mu$, the inequality $G \mu(z) \leq G \varepsilon_{x}(z)$ on $S \mu$ implies the same inequality in $\Omega$.

Ninomiya discussed the equivalence of these principles for symmetric kernels.

TheOREM II. 14. Let $G$ and $\breve{G}$ satisfy the continuity principle. Then $G$ satisfies the domination principle with respect to $N$, if and only if $G$ satisfies the balayage principles with respect to $N$.

This follows immediately from Theorem II. 1.

Theorem II. 15. Let $G$ and $\breve{G}$ satisfy the continuity principle. Then $G$ satisfies the domination principle with respect to $N$, if and only if its adjoint $\breve{G}$ fulfills the following condition:

[A] For $\mu \in \mathfrak{E}_{0}$ and $\nu \in \mathfrak{M}_{0}$, if $\breve{G} \mu \leq \breve{G} \nu$ on $S \mu$, then $\check{N} \mu \leq \check{N} \nu$ in $\Omega$, or equivalently $\breve{G}$ fulfills the condition:

[B] For $\mu \in \varepsilon_{0}$ and $x_{0} \notin S \mu$, if $\breve{G} \mu \leq \breve{G} \varepsilon_{x_{0}}$ on $S \mu$, then $\check{N} \mu \leq \breve{N} \varepsilon_{x_{0}}$ in $\Omega$.

Proof. $G$ satisfies the domination principle with respect to $N \Rightarrow G$ fulfills the condition [A]. Assume that $\breve{G} \mu \leq \breve{G} \nu$ on $S \mu$ for $\mu \in \mathfrak{F}_{0}$ and $\nu \in \mathbb{M}_{0}$, and take an arbitrarily fixed point $x$ in $\Omega$. By Theorem II. 14, th: re exists a positive measure $\varepsilon_{x}^{\prime}$, supported by $S_{\mu}$, such that 


$$
\begin{array}{ll}
G \varepsilon_{x}^{\prime}=N \varepsilon_{x} & G \text {-p.p.p. on } S \mu, \\
G \varepsilon_{x}^{\prime} \leq N \varepsilon_{x} & \text { in } \Omega .
\end{array}
$$

Then

$$
\begin{aligned}
\check{N} \mu(x) & =\int \check{N} \mu d \varepsilon_{x}=\int N \varepsilon_{x} d \mu=\int G \varepsilon_{x}^{\prime} d \mu \\
& =\int \check{G} \mu d \varepsilon_{x}^{\prime} \leq \int \breve{G} \nu d \varepsilon_{x}^{\prime}=\int G \varepsilon_{x}^{\prime} d \nu \\
& \leq \int N \varepsilon_{x} d \nu=\int \check{N} \nu d \varepsilon_{x}=\check{N} \nu(x) .
\end{aligned}
$$

Consequently $\breve{G}$ fufills the condition [A].

$G$ fulfills $[\mathrm{A}] \Longrightarrow G$ fulfills [B]. Evident.

$G$ fulfills $[\mathrm{B}] \Rightarrow G$ satisfies the domination principle with respect to $N$. Assume that $G \mu \leq N \nu$ on $S \mu$ for $\mu \in \mathbb{E}_{0}$ and $\nu \in \mathbb{M}_{0}$ and take a point $x_{0}$ in $\Omega-S \mu$. Then we can take a sequence $\left\{\boldsymbol{u}_{n}\right\}$ of positive finite continuous functions on S $\mu$ such that $u_{n} \nearrow \check{G} \varepsilon_{x_{0}}$. By the existence theorem, there exist positive measures $\lambda_{n}$, supported by $S \mu$, such that

$$
\begin{array}{ll}
\check{G} \lambda_{n} \geq u_{n} & G \text {-p.p.p. on } S \mu, \\
\check{G} \lambda_{n} \leq u_{n} & \text { on } S \lambda_{n} .
\end{array}
$$

Then by the condition $[\mathrm{B}], \breve{N} \lambda_{n} \leq \check{N} \varepsilon_{x_{0}}$ in $\Omega$. Therefore

$$
G \mu\left(x_{0}\right)=\int G \mu d \varepsilon_{x_{0}}=\int \breve{G} \varepsilon_{x_{0}} d \mu=\lim \int u_{n} d \mu
$$

and

$$
\begin{aligned}
\int u_{n} d \mu & \leq \int \breve{G} \lambda_{n} d \mu=\int G \mu d \lambda_{n} \leq \int N \nu d \lambda_{n} \\
& =\int \check{N} \lambda_{n} d \nu \leq \int \check{N} \varepsilon_{x_{v}} d \nu=\int N \nu d \varepsilon_{x_{0}} \\
& =N \nu\left(x_{0}\right) .
\end{aligned}
$$

Consequently $G \mu \leq N \nu$ in $\Omega$. This completes the proof.

Similarly we have

Theorem II. 16. Let $G$ and $\breve{G}$ satisfy the continuity principle. Then $G$ satisfies the elementary domination principle with respect to $N$, if and only if $G$ fulfills the condition:

[C] For a positive measure $\mu \in \mathbb{E}_{0}$ and a point $x_{0} \notin S_{\mu}$, if $\breve{G} \mu \leq \breve{G} \varepsilon_{x_{0}}$ on $S_{\mu}$, then $\check{N} \mu \leq \check{N} \varepsilon_{x_{0}}$ in $\Omega-S \mu$. 
Remark. Evidently $G$ satisfies the elementary domination principle with respect to $N$, if $G$ satisfies the domination principle with respect to $N$. But the converse is not true; there is a simple counter-example. Thus we ask a question: under what condition for $G$ and/or $N$ does the elementary domination principle with respect to $N$ imply the domination principle with respect to $N$ ? We can give only the following answer. Needless to say, the implication holds if $N=G$ or $N=1$ and $G$ and $\breve{G}$ satisfy the continuity principle.

ThEOREM II. 17. Let $G$ and $\breve{G}$ satisfy the continuity principle. If $G(x, x)=$ $+\infty$ at every point $x \in \Omega$, then the following implication is true for $G$ : the elementary domination principle with respect to $N \Rightarrow$ the domination principle respect with to $N$.

Proof. It is easy to see that for a compact set $K$ and a point $x \notin K$, there exists a positive measure $\lambda$, supported by $K$, such that

$$
\begin{array}{ll}
G \lambda=N \varepsilon_{x} & G \text {-p.p.p. on } K, \\
G \lambda \leq N \varepsilon_{x} & \text { in } \Omega,
\end{array}
$$

if $G$ satisfies the elementary domination principle with respect to $N$. From this remark follows our theorem.

\section{REFERENCES}

[1] P. Alexandroff and H. Hopf: Topologie I, Berlin, 1935.

[2] M. Brelot and G. Choquet: Le théorème de convergence en théorie du potentiel, Jour. Madras Univ., B. 27 (1957), 277-286.

[3] H. Cartan and J. Deny: Le principe du maximum en théorie du potentiel et la notion de fonction surharmonique, Acta Sci. Math. Szeged, 12 (1950), 81-100.

[ 4] G. Choquet and J. Deny: Modèles finis en théorie du potentiel, Jour. Anal. Math., 5 (1956-57), 77-135.

[5] J. Deny: Les deux aspects de la théorie du potentiel, Sém. Bourbaki, (1957), no. 148,18 pp.

[6] J. Deny: Les principes du maximum en théorie du potentiel, Sém. Brelot-ChoquetDeny, 6e année (1962), no. 10, 8 pp.

[ 7 ] O. Frostman: Potentiel d'équilibre et capacité des ensembles avec quelques applications à la théorie des fonctions, Thèse, Lund, (1935), 118 pp.

[8] G. A. Hunt: Markoff processes and potentials II, Illinois Jour. Math., 1 (1957), 316369.

[9] S. Kametani: Positive definite integral quadratic forms and generalized potentials, Proc. Imp. Acad. Japan, 20 (1944), 7-14.

[10] M. Kishi: Unicity principles in the potential theory, Osaka Math. Jour., 13 (1961), 41-74. 
[11] M. Kishi: Note on balayage and maximum principles, Proc. Japan Acad., 39 (1963), 415-418.

[12] N. Ninomiya: Sur le théorème du balayage et le théorème d'équilibre, Jour. Inst. Polytech. Osaka City Univ., 6 (1955), 83-91.

[13] N. Ninomiya: Etude sur la théorie du potentiel pris par rapport au noyau symétrique, ibid. 8 (1957), 147-179.

[14] N. Ninomiya: Sur le problème du balayage généralisé, Jour. Math. Osaka City Univ., 12 (1961), 115-138.

[15] N. Ninomiya: Sur un principe du maximum dans la théorie du potentiel, ibid. 12 (1961), 139-143.

[16] M. Ohtsuka: On potentials in locally compact spaces, Jour. Sci. Hiroshima Univ Ser. A-I, 25 (1961), 135-352. 